\title{
Search for SM Higgs decaying to WW and WW production cross section measurement at CMS
}

\author{
Lara Lloret Iglesias ${ }^{1}$ \\ Universidad de Oviedo \\ Departamento de Física \\ C/ Jesús Arias de Velasco \\ 33005 Oviedo, Spain \\ E-mail: lara@cern. ch
}

A search for the standard model Higgs boson decaying to $\mathrm{W}^{+} \mathrm{W}^{-}$in pp collisions at $\sqrt{s}_{\mathrm{s}}=8 \mathrm{TeV}$ is reported. The event sample corresponds to an integrated luminosity of $5.1 \pm 0.2 \mathrm{fb}^{-1}$, collected by the CMS detector at the LHC. The $\mathrm{W}^{+} \mathrm{W}^{-}$candidates are selected in events with two opposite charged leptons and large missing transverse momentum. Upper limits on the Higgs boson production relative to the standard model Higgs expectation are derived. The standard model Higgs boson is excluded in the mass range $129-520 \mathrm{GeV}$ at $95 \%$ confidence level when these results are combined with the analysis performed at $\sqrt{s}=7 \mathrm{TeV}$. A small excess of events is observed for hypothetical low Higgs boson masses which makes the observed limits weaker than the expected ones under the null hypothesis. This document also reports on a measurement of the $\mathrm{W}^{+} \mathrm{W}^{-}$production cross section in pp collisions at $\sqrt{\mathrm{s}}=8 \mathrm{TeV}$. The data were collected at the LHC with the CMS detector, and correspond to an integrated luminosity of $3.54 \mathrm{fb}^{-1}$. The $\mathrm{W}^{+} \mathrm{W}^{-}$cross section is measured to be $69.9 \pm 2.8$ (stat.) \pm 5.6 (syst.) \pm 3.1 (lumi.) pb.

1

Lara Lloret Iglesias 
36th International Conference on High Energy Physics

July 4-11, 2012

Melbourne, Australia 


\section{Introduction}

The search for the Higgs boson in the $\mathrm{H} \rightarrow \mathrm{W}^{+} \mathrm{W}^{-} \rightarrow 212 v$ final state, where 1 is a charged lepton, electron or muon, and $v$ a neutrino, is presented in this document. Previous results in this final state were published by CMS based on the 2010 and 2011 datasets $[1,2]$, excluding the presence of the Higgs boson in the mass range $129-270 \mathrm{GeV}$. The search discussed here is performed over the mass range $110-600 \mathrm{GeV}$, and the data sample corresponds to $5.1 \pm 0.2 \mathrm{fb}^{-1}$ of integrated luminosity collected in 2012 at a center-of-mass energy of $8 \mathrm{TeV}$. Finally, the $\sqrt{\mathrm{s}}_{\mathrm{s}}=7 \mathrm{TeV}$ data sample is added to the analysis to obtain the combined 2011 and 2012 results. This conference note reports a measurement of the $\mathrm{W}^{+} \mathrm{W}^{-}$ production cross section in pp collisions at $\sqrt{\mathrm{s}}=8 \mathrm{TeV}$, performed on a dataset corresponding to an integrated luminosity of $3.54 \mathrm{fb}^{-1}$, collected during 2012 .

\subsection{W+W- events selection}

The search strategy for $\mathrm{H} \rightarrow \mathrm{W}^{+} \mathrm{W}^{-}$is based on the final state in which both $\mathrm{W}$ bosons decay leptonically, resulting in a signature with two isolated, oppositely charged, high $\mathrm{p}_{\mathrm{T}}$ leptons (electrons or muons) and large missing transverse momentum, $\mathrm{E}_{\mathrm{T}}{ }^{\text {miss }}$, due to the undetected neutrinos. To improve the signal sensitivity, the events are separated by jet multiplicity into three mutually exclusive categories, which are characterized by different signal yields and signal-to-background ratios. In the following we call these the 0-jet, 1-jet and 2-jet bins. Events with more than 2 jets are not considered. Furthermore, the search strategy splits signal candidates into three final states denoted by: $\mathrm{e}^{+} \mathrm{e}^{-}, \mu^{+} \mu^{-}$, and $\mathrm{e}^{ \pm} \mu^{\mp}$. The bulk of the signal arises through direct $\mathrm{W}$ decays to electrons or muons of opposite charge, where the small contribution proceeding through an intermediate $\tau$ lepton is implicitly included. The events are selected by triggers which require the presence of one or two high- $\mathrm{p}_{\mathrm{T}}$ electrons or muons. The trigger efficiency for signal events that pass the full event selection is measured to be above $97 \%$ in the $\mu^{+} \mu$ final state, and about $98 \%$ in the $\mathrm{e}^{+} \mathrm{e}^{-}$and $\mathrm{e}^{ \pm} \mu^{\mp}$. final states for a Higgs boson mass of about $125 \mathrm{GeV}$. The trigger efficiencies increase with the Higgs boson mass.

Two oppositely charged lepton candidates are required, with $\mathrm{p}_{\mathrm{T}}>20 \mathrm{GeV}$ for the leading lepton $\left(\mathrm{p}_{\max \mathrm{T}}\right)$ and $\mathrm{p}_{\mathrm{T}}>10 \mathrm{GeV}$ for the trailing lepton ( $\mathrm{p}_{\min \mathrm{T}}$ ). Only electrons (muons) with $|\eta|<2.5$ (2.4) are considered in the analysis.

Muon candidates are identified using a selection similar to the one described in [2]. Muons are required to be isolated to distinguish between muon candidates from $\mathrm{W}$-boson decays and those from QCD background processes, which are usually in or near jets.

The lepton candidates are required to originate from the primary vertex of the event, which is chosen as the vertex with the highest $\sum \mathrm{p}_{\mathrm{T}}{ }^{2}$, where the sum runs over the tracks associated with the vertex, including the tracks associated with the leptons.

Jets are reconstructed using the anti- $\mathrm{k}_{\mathrm{T}}$ clustering algorithm [3] with distance parameter $\Delta \mathrm{R}=$ 0.5. A similar correction as for the lepton isolation is applied to account for the contribution to the jet energy from the pile-up. Jet energy corrections are applied as a function of the jet $E_{T}$ and $\eta[4]$.

The properties of the hard jets are modified by particles from pile-up interactions. A combinatorial background arises from low- $\mathrm{p}_{\mathrm{T}}$ jets from pile-up interactions which get clustered into high- $\mathrm{p}_{\mathrm{T}}$ jets. A multivariate selection is applied to separate jets from the primary interaction from those reconstructed due to energy deposits associated with pile-up.

Events are classified according to the number of selected jets with $\mathrm{E}_{\mathrm{T}}>30 \mathrm{GeV}$ and $|\eta|<4.7$. In addition to high momentum isolated leptons and minimal jet activity, missing transverse momentum is present in signal events but generally not in background. 
It is equal to the component of $\mathrm{E}_{\mathrm{T}}{ }^{\text {miss }}$ transverse to the nearest lepton if the difference in azimuth between this lepton and the $\mathrm{E}_{\mathrm{T}}{ }^{\text {miss }}$ vector is less than $\pi / 2$. If there is no lepton within $\pi / 2$ of the direction of $\mathrm{E}_{\mathrm{T}}^{\text {miss }}$ in azimuth, $\mathrm{E}_{\mathrm{T}}{ }^{\text {miss }}$ is used directly.

The $\mathrm{E}_{\mathrm{T}}{ }^{\text {miss }}$ reconstruction makes use of event reconstruction via the particle-flow technique [5].Since the projected $E_{T}$ miss resolution is degraded by pile-up, the minimum of two $E_{T}$ miss observables is used: the first includes all reconstructed particles in the event, while the second uses only the charged particles associated with the primary vertex. Events with projected $\mathrm{E}_{\mathrm{T}}$ miss above $20 \mathrm{GeV}$ are selected for the analysis.

To suppress the top-quark background, a top tagging technique based on soft-muon and b-jet tagging [6,7] is applied. The first method is designed to veto events containing muons from b-quarks coming from top-quark decays. The second method uses b-jet tagging algorithm which looks for tracks with large impact parameter within jets. The rejection factor for the top quark background is about $50 \%$ in the 0 -jet category and above $80 \%$ for events with at least one jet passing the selection criteria. A minimum dilepton transverse momentum of $45 \mathrm{GeV}$ is required to reduce the $\mathrm{W}+$ jets background.

To reduce the background from $\mathrm{WZ}$ production, any event that has a third lepton passing the identification and isolation requirements is rejected.

In order to suppress the Drell-Yan background, a few additional cuts are applied in the sameflavor final states. First, the resonant component of the Drell-Yan production is rejected by requiring a dilepton mass outside a $30 \mathrm{GeV}$ window centered on the $\mathrm{Z}$ pole. Then, the remaining off-peak contribution is suppressed by exploiting different $\mathrm{E}_{\mathrm{T}}{ }^{\text {miss }}$-based approaches depending on the number of jets and the Higgs mass hypothesis. At large Higgs masses $\left(m_{H}>140 \mathrm{GeV}\right)$, signal events are associated with large $\mathrm{E}_{\mathrm{T}}{ }^{\text {miss }}$ and, thus, to suppress the Drell-Yan background it is sufficient to require the minimum of the two projected $\mathrm{E}_{\mathrm{T}}{ }^{\text {miss }}$ variables to be greater than 45 $\mathrm{GeV}$. On the contrary, in low Higgs mass analyses $\left(\mathrm{m}_{\mathrm{H}}<140 \mathrm{GeV}\right)$ it is more difficult to separate the signal from the Drell-Yan background; therefore, in this case, a dedicated multivariate selection combining missing transverse momentum, kinematic and topological variables, is used to reject Drell-Yan events and maximize the surviving signal yield. A third approach is employed in events with two jets: here, the dominant source of fake $\mathrm{E}_{\mathrm{T}}{ }^{\text {miss }}$ is the mismeasurement of the hadronic recoil and the optimal performance is obtained by simply requiring $\mathrm{E}_{\mathrm{T}}{ }^{\text {miss }}>45 \mathrm{GeV}$. Finally, the momenta of the dilepton system and of the most energetic jet must not be back-to-back in the transverse plane. These selections effectively reduce the Drell-Yan background by three orders of magnitude, while rejecting less than $50 \%$ of the signal. To estimate the WW production cross section we require both leptons to have $\mathrm{p}_{\mathrm{T}}>$ $20 \mathrm{GeV}$.

\section{$1.2 \mathrm{H} \rightarrow \mathrm{W}^{+} \mathrm{W}^{-}$search strategy}

To enhance the sensitivity to a Higgs boson signal, a cut-based approach is chosen for the final "Higgs" selection. Because the kinematics of signal events change as a function of the Higgs mass, separate optimizations are performed for different $m_{H}$ hypotheses.

The extra requirements, designed to optimize the sensitivity for a SM Higgs boson, are placed on $\mathrm{p}_{\operatorname{maxT}}, \mathrm{p}_{\mathrm{min} \mathrm{T}}, \mathrm{m}_{\mathrm{ll}}, \Delta \phi_{l l}$ and the transverse mass $\mathrm{m}_{\mathrm{T.}}$. The $\Delta \phi_{l l}$ and $\mathrm{m}_{l l}$ distributions in the 0 -jet and 1-jet categories, for a $\mathrm{m}_{\mathrm{H}}=125 \mathrm{GeV}$ SM Higgs boson and for the main backgrounds are shown in Figures 1 and 2, respectively.

The 2-jet category is mainly sensitive to the vector boson fusion (VBF) production mode, whose cross section is roughly ten times smaller than that of the gluon-gluon fusion mode. The $\mathrm{H} \rightarrow \mathrm{W}^{+} \mathrm{W}^{-}$events from VBF production are characterized by a pair of energetic forwardbackward jets and very little hadronic activity in the rest of the event. Events passing the $\mathrm{W}^{+} \mathrm{W}^{-}$ criteria are further required to satisfy $\mathrm{p}_{\mathrm{T}}>30 \mathrm{GeV}$ for both leading jets, with no jets above this threshold present in the pseudorapidity region between the two leading jets. The two leptons are 
required to be within the pseudorapidity region defined by the two jets. To reject the main background from top-quark decays, two additional requirements are applied to the two jets, $\mathrm{j}_{1}$ and $\mathrm{j}_{2}:\left|\Delta \eta\left(\mathrm{j}_{1}, \mathrm{j}_{2}\right)\right|>3.5$ and $\mathrm{m}_{\mathrm{j} 1 \mathrm{j} 2}>450 \mathrm{GeV}$. In addition, $\mathrm{m}_{\mathrm{T}}$ is required to be larger than 30 $\mathrm{GeV}$ and smaller than the Higgs mass hypothesis. Finally, a $\mathrm{m}_{\mathrm{H}}$ dependent upper limit on the dilepton mass is applied.

\subsection{Background estimation}

The W+ jets and QCD multijet backgrounds arise from leptonic decays of heavy quarks, hadrons misidentified as leptons, and electrons from photon conversion. The estimate of these contributions is derived directly from data using a control sample of events in which one lepton passes the standard criteria and the other does not, but instead satisfies a relaxed set of requirements ("loose" selection), resulting in a "tight-fail" sample. The efficiency, $\varepsilon_{\text {loose }}$, for a jet that satisfies the loose selection to pass the tight selection is determined using data from an independent multijet event sample dominated by non-prompt leptons, and parameterized as a function of $\mathrm{p}_{\mathrm{T}}$ and $\eta$ of the lepton. The background contamination is then estimated using the events of the "tight-fail" sample weighted by $\varepsilon_{\text {loose }} /\left(1-\varepsilon_{\text {loose }}\right)$. The systematic uncertainties from the efficiency determination dominate the overall uncertainty of this method, which is estimated to be about $36 \%$.

The normalization of the top-quark background is estimated from data as well by counting the number of top-tagged $\left(\mathrm{N}_{\text {tagged }}\right)$ events and applying the corresponding top-tagging efficiency. The top-tagging efficiency ( $\left.\varepsilon_{\text {top tagged }}\right)$ is measured with a control sample dominated by tt and tW events, which is selected by requiring a b-tagged jet. The residual number of top events $\left(\mathrm{N}_{\text {not tagged }}\right)$ in the signal region is given by: $\mathrm{N}_{\text {not tagged }}=\mathrm{N}_{\text {tagged }} \times\left(1-\varepsilon_{\text {top tagged }}\right) / \varepsilon_{\text {top tagged }}$.

Background sources from non-top events are subtracted by estimating the misidentification probability from data control samples. The main uncertainty comes from the statistical uncertainty in the control sample and from the systematic uncertainties related to the measurement of $\varepsilon_{\text {top tagged }}$. The uncertainty is about $20 \%$ in the 0 -jet category and about $5 \%$ in the 1 -jet category.

For the low-mass $\mathrm{H} \rightarrow \mathrm{W}^{+} \mathrm{W}^{-}$signal region, $\mathrm{m}_{\mathrm{H}} \leq 200 \mathrm{GeV}$, the non-resonant $\mathrm{W}^{+} \mathrm{W}^{-}$ contribution is estimated from data. This contribution is measured using events with a dilepton mass larger than $100 \mathrm{GeV}$, where the Higgs boson signal contamination is negligible, and a simulation is used to extrapolate into the signal region. The total uncertainty is about $10 \%$. For larger Higgs boson masses there is a large overlap between the non-resonant $\mathrm{W}^{+} \mathrm{W}^{-}$and Higgs boson signal, and simulation is used for the estimation.

The $\mathrm{Z} / \gamma^{*} \rightarrow 1^{+} 1^{-}$contribution to the $\mathrm{e}^{+} \mathrm{e}^{-}$and $\mu^{+} \mu$ final states is based on extrapolation from the observed number of events with a dilepton mass within $\pm 7.5 \mathrm{GeV}$ of the $\mathrm{Z}$ mass, where the residual background in that region is subtracted using $\mathrm{e}^{ \pm} \mu^{\mp}$ events. The extrapolation to the signal region is performed using the simulation, and the results are cross-checked with data, using the same algorithm and subtracting the background in the $\mathrm{Z}$ pole region which is estimated from $\mathrm{e}^{ \pm} \mu^{\mp}$ events. The largest uncertainty in the estimate is the statistical uncertainty of the control sample, which is about $20 \%$ to $50 \%$. The $\mathrm{Z} / \gamma^{*} \rightarrow \tau^{+} \tau^{-}$contamination is estimated using $\mathrm{Z} / \gamma^{*} \rightarrow \mathrm{e}^{+} \mathrm{e}^{-}$and $\mathrm{Z} / \gamma^{*} \rightarrow \mu^{+} \mu^{-}$events selected in data, where the leptons are replaced with simulated $\tau$ decays, thus providing a better description of the experimental conditions with respect to the full simulation of the process $\mathrm{Z} / \gamma^{*} \rightarrow \tau^{+} \tau^{-}$.

Finally, to estimate the $\mathrm{W}^{*}$ background contribution from asymmetric virtual photon decays, where one lepton escapes detection. To obtain the normalization scale of the simulated events a control sample of high purity $\mathrm{W} \gamma^{*}$ events with three reconstructed leptons is defined and compared to the simulation prediction. A measured factor of $1.6 \pm 0.5$ with respect to the leading order cross section is found. 
Other minor backgrounds from WZ, ZZ (when the two selected leptons come from different bosons) and $\mathrm{W} \gamma$ are estimated from simulation.

\subsection{Results}

\subsection{1 $\mathrm{H} \rightarrow \mathrm{W}+\mathrm{W}$ - searches}

After applying the mass-dependent Higgs selection, upper limits are derived on the ratio of the product of the Higgs boson production cross section and the $\mathrm{H} \rightarrow \mathrm{W}^{+} \mathrm{W}^{-}$branching fraction, $\sigma_{\mathrm{H}} \times \mathrm{BR}\left(\mathrm{H} \rightarrow \mathrm{W}^{+} \mathrm{W}^{-}\right)$, and the $\mathrm{SM}$ Higgs expectation, $\sigma \sigma_{\mathrm{SM}}$.

To compute the upper limits the modified frequentist construction CLs [8-10] is used. The number of events in each bin is modeled as a Poisson random variable, whose mean value is the sum of the contributions from signal and background processes. All the sources of systematic uncertainties [1,2] are considered. The 95\% CL observed and expected median upper limits are shown in Fig. 1.

The $8 \mathrm{TeV}$ analysis excludes the presence of a Higgs boson with mass in the range 135-198 $\mathrm{GeV}$ at $95 \% \mathrm{CL}$, while the expected exclusion limit in the hypothesis of background only is 128-250 GeV. With the addition of the $7 \mathrm{TeV}$ analysis, a Higgs boson with mass in the range $129-520 \mathrm{GeV}$ is excluded at $95 \% \mathrm{CL}$, while the expected exclusion limit for the background only hypothesis is in the range $122-450 \mathrm{GeV}$. The observed (expected) upper limits are about 2.2 (1.2) times the $\mathrm{SM}$ expectation for $\mathrm{m}_{\mathrm{H}}=125 \mathrm{GeV}$.

A small excess of events is observed for hypothetical low Higgs boson masses, which makes the observed limits weaker than the expected ones. Due to the poor mass resolution of this approach channel the excess extends over a large mass range.

\subsubsection{WW production cross section}

Counting the number of events in the signal region, the $\mathrm{W}^{+} \mathrm{W}^{-}$yield is calculated by subtracting the estimated contributions of the various SM background processes. The signal efficiency times acceptance averaged over all lepton flavors including $\tau \mathrm{s}$ is found to be $(3.22 \pm 0.22$ (total))\%.

The total background yield is $275.2 \pm 14.9$ (stat.) \pm 31.2 (syst.) events and the total number of events observed is 1111 . Using the $\mathrm{W} \rightarrow 1 v$ branching ratio of $(0.1080 \pm 0.0009)$ from Ref. [11], the $\mathrm{W}^{+} \mathrm{W}^{-}$production cross section in pp collision data at $\sqrt{\mathrm{s}}=8 \mathrm{TeV}$, is calculated to be:

$$
\sigma_{\mathrm{ww}}=69.9 \pm 2.8 \text { (stat.) } \pm 5.6 \text { (syst.) } \pm 3.1 \text { (lumi.) pb. }
$$

The statistical uncertainty is due to the total number of observed events. The systematic uncertainty includes both the statistical and systematic uncertainties on the background prediction, as well as the uncertainty on the signal efficiency. This measurement is consistent with the SM expectation of $57.3_{-1.6}^{+2.4} \mathrm{pb}$ [12]. The difference between the measured and the theoretical value is $12.6 \pm 7.3 \mathrm{pb}$, equivalent to $(22 \pm 13) \%$ of the theoretical value. Experimental and theoretical uncertainties have been added in quadrature. 

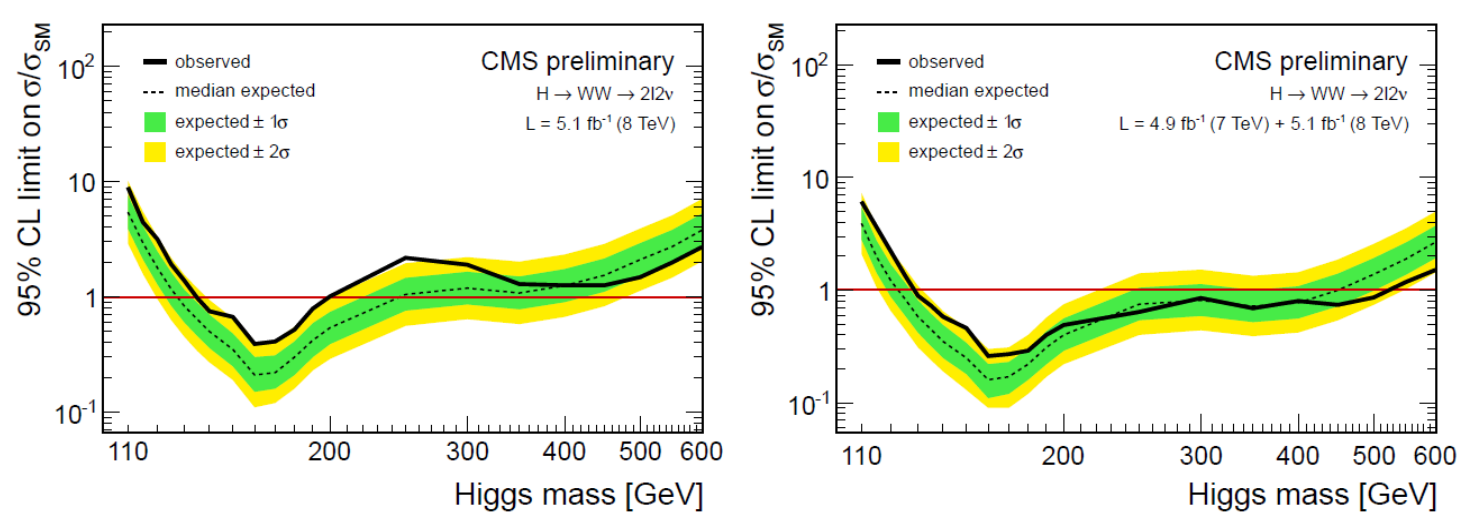

Figure 1. Expected and observed 95\% CL upper limits on the cross section times branching fraction, $\sigma_{\mathrm{H}} \times \mathrm{BR}\left(\mathrm{H} \rightarrow \mathrm{W}^{+} \mathrm{W}^{-}\right)$, relative to the $\mathrm{SM}$ Higgs expectation, using the $8 \mathrm{TeV}$ data only (left) and the combined $7 \mathrm{TeV}$ and $8 \mathrm{TeV}$ data (right). Results are obtained using the CLs approach.

\section{References}

[1] CMS Collaboration, "Measurement of WW Production and Search for the Higgs Boson in $\mathrm{pp}$ Collisions at $\sqrt{\mathrm{s}}=7$ TeV", Phys. Lett. B 699 (2011) 25, doi:10.1016/j.physletb.2011.03.056.

[2] CMS Collaboration, "Search for the standard model Higgs boson decaying to a W pair in the fully leptonic final state in pp collisions at $\sqrt{\mathrm{s}}=7 \mathrm{TeV}$ ", Phys. Lett. B 710 (2012) 91, doi:10.1016/j.physletb.2011.03.056

[3] M. Cacciari and G. P. Salam and G. Soyez, "The anti-k jet clustering algorithm", JHEP 04 (2008) 063, doi:10.1088/1126-6708/2008/04/063, arXiv:0802.1189.

[4] CMS Collaboration, "Determination of Jet Energy Calibration and Transverse Momentum Resolution in CMS", JINST 6 (2011) 11002, doi:10.1088/17480221/6/11/P11002.

[5] CMS Collaboration, "Particle-Flow Event Reconstruction in CMS and Performance for Jets, Taus, and $\mathrm{E}_{\mathrm{T}}{ }^{\text {miss }}$, CMS Physics Analysis Summary CMS-PAS-PFT-09-001, (2009).

[6] CMS Collaboration, "Algorithms for b Jet Identification in CMS", CMS Physics Analysis Summary CMS-PAS-BTV-09-001, (2009).

[7] CMS Collaboration, "Commissioning of b-jet identification with pp collisions at $\sqrt{\mathrm{s}}_{\mathrm{s}}=7$ TeV”, CMS Physics Analysis Summary CMS-PAS-BTV-10-001, (2010).

[8] A. L. Read, "Presentation of search results: the CLs technique", J. Phys. G: Nucl. Part. hys. 28 (2002) 2693, doi:10.1088/0954-3899/28/10/313.

[9] T. Junk, "Confidence level computation for combining searches with small statistics", Nucl. Instrum. Meth. A434 (1999) 435, doi:10.1016/S0168-9002(99)00498-2. 
[10] ATLAS and CMS Collaborations, LHC Higgs Combination Group, "Procedure for the LHC Higgs boson search combination in Summer 2011", ATL-PHYS-PUB/CMS NOTE 2011-11, 2011/005, (2011).

[11] K. Nakamura et al. (Particle Data Group), "Review of particle physics", J. Phys. G37 (2010) 075021, doi:10.1088/0954-3899/37/7A/075021.

[12] J. M. Campbell, R. Ellis, and C. Williams, "Vector boson pair production at the LHC", JHEP 1107 (2011) 018, doi:10.1007/JHEP07(2011)018, arXiv:1105.0020. 